Review

\title{
Advances in Lipid Nanoparticles for siRNA Delivery
}

\author{
Yuen Yi C. Tam *, Sam Chen and Pieter R. Cullis \\ Department of Biochemistry and Molecular Biology, University of British Columbia, \\ 2350 Health Sciences Mall, Vancouver, B.C. V6T 1Z3, Canada; \\ E-Mails: s.chen@alumni.ubc.ca (S.C.); pieterc@mail.ubc.ca (P.R.C.) \\ * Author to whom correspondence should be addressed; E-Mail: tam7@mail.ubc.ca; \\ Tel.: +1-604-822-6263; Fax: +1-604-822-4843.
}

Received: 1 July 2013; in revised form: 4 September 2013 / Accepted: 12 September 2013 / Published: 18 September 2013

\begin{abstract}
Technological advances in both siRNA (small interfering RNA) and whole genome sequencing have demonstrated great potential in translating genetic information into siRNA-based drugs to halt the synthesis of most disease-causing proteins. Despite its powerful promises as a drug, siRNA requires a sophisticated delivery vehicle because of its rapid degradation in the circulation, inefficient accumulation in target tissues and inability to cross cell membranes to access the cytoplasm where it functions. Lipid nanoparticle (LNP) containing ionizable amino lipids is the leading delivery technology for siRNA, with five products in clinical trials and more in the pipeline. Here, we focus on the technological advances behind these potent systems for siRNA-mediated gene silencing.
\end{abstract}

Keywords: lipid nanoparticle; siRNA; ionizable amino lipid; pKa; PEG lipid; targeting

\section{Introduction}

RNA interference (RNAi), since its discovery in the 1990s, has rapidly become the most powerful tool for studying functional genomics and validating gene targets in vitro and in vivo, as well as developing gene-specific medicines [1,2]. The small interfering RNA (siRNA) is a double-stranded oligonucleotide composed of typically 19-25 base pairs. It binds to the RNA-induced silencing complex (RISC) and guides the cleavage of the targeted mRNA sequence. Advances in design algorithms and nucleotide chemistry for siRNA, combined with the completion of the human genome and advances in whole genome sequencing technologies, have presented exciting opportunities to 
rapidly translate genomic information (such as whole genome sequencing of tumour tissue to identify causal genes) into siRNA-based therapeutics to block the production of disease-causing proteins [2-4]. siRNAs can be designed to target most genes in the genome, from one gene at a time to several genes simultaneously, vastly expanding the number of "druggable" targets.

The major limitation of siRNA use in the clinic is the development of safe and effective systemic delivery systems. "Naked" siRNA is highly unstable and rapidly degraded by nucleases in biological fluids, does not accumulate in target tissues and cannot readily cross target cell membranes to access its cytoplasmic site of action [5,6]. Although there are significant advancements in siRNA design and chemical modifications that both increase stability and reduce immunogenicity [7-10], there is still a need for efficient cellular uptake and target site accumulation. Appropriate delivery vehicles are therefore essential for realizing the potential of siRNA technology. Delivery strategies include both polymer- and lipid-based systems [11,12]. Among all delivery platforms, lipid nanoparticles (LNPs) containing ionizable amino lipids represent the leading system for intravenously (i.v.) administered RNAi-based therapeutics. It has been shown that LNP siRNA systems are capable of hepatocyte gene silencing in vivo at doses as low as $0.005 \mathrm{mg} / \mathrm{kg}$ in animal models following i.v. injection, which is currently the worldwide gold standard for siRNA-based therapeutics [13,14]. Five LNP siRNA formulations are currently in various stages of clinical development. Alnylam Pharmaceuticals has three products in their pipeline, which target transthyretin (TTR) for the treatment of TTR-mediated amyloidosis (ALN-TTR02), vascular endothelial growth factor (VEGF) and kinesin spindle protein (KSP) for hepatocellular carcinoma (ALN-VSP), and proprotein convertase subtilisin/kexin type 9 (PCSK9) for hypercholesterolemia (ALN-PCS). In addition, Tekmira Pharmaceuticals has developed TKM-PLK1 that targets polo-like kinase 1 (PLK1) for solid tumors and TKM-Ebola to treat Ebola virus infection. All five products show very safe clinical profiles and promising activity. They are described in detail in other reviews [15-19] and their respective company websites (http://www.alnylam.com/ Programs-and-Pipeline/index.php and http://www.tekmirapharm.com/Programs/Products.asp). This review is focused on the technological bases behind these advanced LNP systems.

\section{LNP Formulation: Composition, Structure and Size}

The advanced LNP siRNA systems are lipid-based particles with diameters less than $100 \mathrm{~nm}$ as measured by dynamic light scattering and cryo-transmission electron microscopy (cryoTEM). They are composed of an ionizable amino lipid (e.g., heptatriaconta-6,9,28,31-tetraen-19-yl 4-(dimethylamino)butanoate, DLin-MC3-DMA [14]), a phosphatidylcholine (1,2-distearoyl-sn-glycero-3phosphocholine, DSPC), cholesterol and a coat lipid (polyethylene glycol-dimyristolglycerol, PEG-DMG) at molar ratio of 50:10:38.5:1.5 (Figure 1). CryoTEM revealed that LNP systems formed by mixing an ethanol stream containing the lipid mixture with an aqueous stream containing the siRNA, by either a T-tube [20] or microfluidic-based mixing [21,22], have an electron-dense core instead of the less dense aqueous core characteristic of vesicular structures [21,23]. Indeed, the LNP siRNA systems have an interior lipid core containing siRNA complexed with ionizable cationic lipid, as shown by the absence of ${ }^{31} \mathrm{P}$ NMR signal from free phosphorothioate in the siRNA and the complete protection of siRNA degradation by external RNases [21]. Computer simulation of the self-assembly of lipids suggests a nanostructured core in which siRNA is located in internal inverted micelles complexed with ionizable 
amino lipid (Figure 2). Furthermore, DSPC interacts with the siRNA phosphate through its choline group, cholesterol is dispersed about evenly between the core and surface, and the polyethylene glycol-lipid conjugate (PEG-lipid) is distributed predominantly on the surface. It is postulated that the rapid mixing of lipid components with the siRNA allows for the formation of nucleating structures composed of siRNA and ionizable amino lipid. As the polarity of the mixing environment increases, coating of the nucleating structures by the remaining lipids occurs until they reach their solubility limits, thus forming the final LNP siRNA structure [21]. The size of LNP siRNA systems made by the microfluidic technique is dictated by the PEG-lipid content [22]. As the amount of PEG-lipid increases from $0.5 \mathrm{~mol} \%$ to $5 \mathrm{~mol} \%$, LNP diameter decreases from approximately $100 \mathrm{~nm}$ to $25 \mathrm{~nm}$. siRNA encapsulation efficiencies are unaffected and remain greater than 95\% [21,22].

Figure 1. Lipid nanoparticle (LNP) siRNA systems are typically composed of ionizable amino lipids, phosphatidylcholine lipids, cholesterol and polyethylene glycol-lipid conjugate (PEG-lipids). Structure of heptatriaconta-6,9,28,31-tetraen-19-yl 4-(dimethylamino)butanoate (DLin-MC3-DMA), 1,2-distearoyl-sn-glycero-3-phosphocholine (DSPC), polyethylene glycol-dimyristolglycerol (PEG-DMG) and cholesterol is shown.

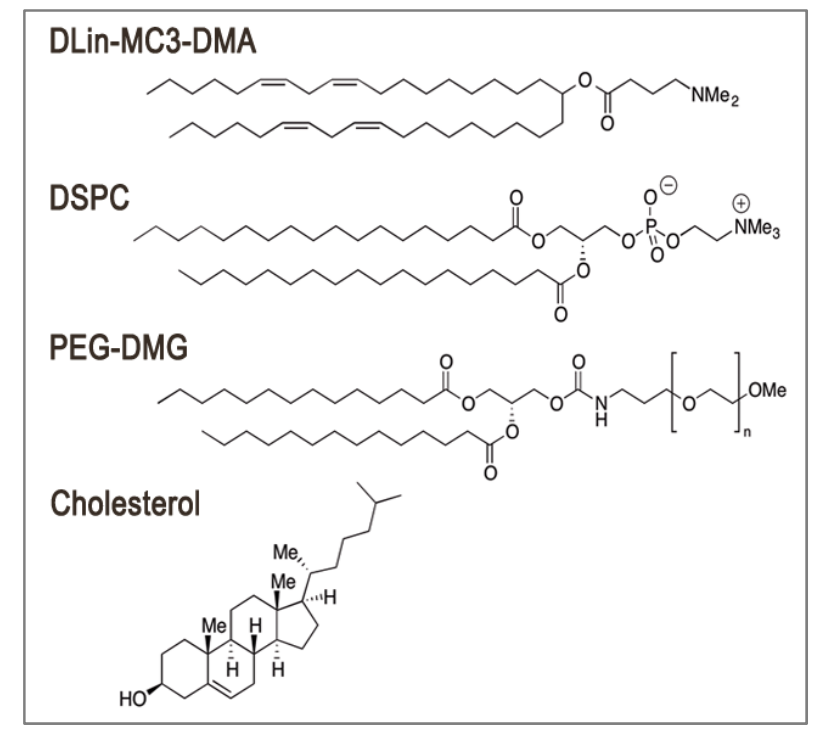

Figure 2. A schematic of LNP siRNA showing a nanostructured core.

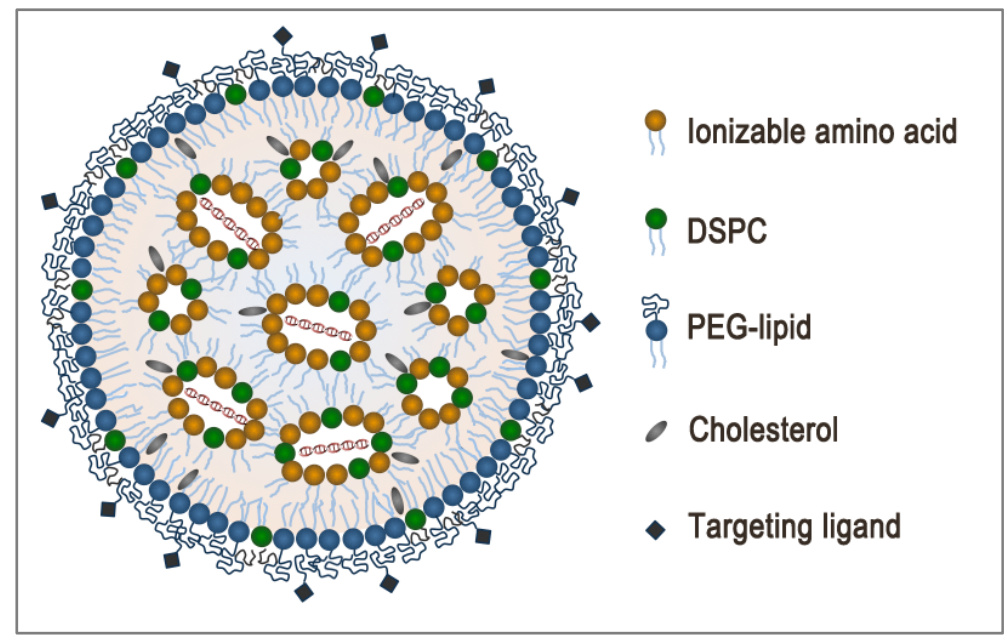




\section{Encapsulation and Intracellular Release: Dual Function of Ionizable Amino Lipids}

A major advancement in the design of ionizable cationic lipids was the modulation of their apparent acid dissociation constant $\left(\mathrm{pK}_{\mathrm{a}}\right) \cdot \mathrm{pK}_{\mathrm{a}}$ values of 7 and lower have been shown to be critical for encapsulation of nucleic acids and in vivo activity [24,25]. In environments where the $\mathrm{pH}$ is below the $\mathrm{pK}_{\mathrm{a}}$ of the ionizable lipid (e.g., $\mathrm{pH} 4.0$ ), the amino group is protonated and interacts with the negatively charged nucleic acids, thereby promoting the self-assembly of the formulation components into nanoparticles encapsulating the siRNA. In physiological environments where the $\mathrm{pH}$ is above the $\mathrm{pK}_{\mathrm{a}}$ of the ionizable lipid (e.g., $\mathrm{pH}$ 7.4), the surface of the LNP has an almost neutral charge, which improves circulation and reduces toxicity. Subsequently in the acidic environment of endosomes, the amino group of the ionizable lipid is positively charged and associates with the anionic endosomal lipids. This interaction enables the destabilization of the endosomal membranes and promotes the release of siRNA into the cytosol [26,27].

The first ionizable amino lipid that was used for nucleic acid encapsulation was 1,2-dioleoyl-3dimethyaminopropane (DODAP), which has a pKa of 6.6-7 and one double bond in each of its acyl chains $[24,25,28]$. Subsequent work focused on the impact of the number of double bonds in the acyl chain demonstrated that ionizable lipids containing fully saturated acyl chains showed no silencing of luciferase activity in vitro, whereas ionizable lipids containing two or three double bonds per acyl chain showed enhanced silencing activity [29]. Encapsulation efficiencies of siRNA seemed to be compromised using ionizable lipids that contain three double bonds per acyl chain. Therefore, the linoleyl lipid has become the acyl chain of choice in subsequent ionizable lipid development.

A structure-activity relationship guided synthesis and screening of a large number of ionizable lipids with various types of linkers connecting the amino group and the acyl chains. These studies have identified DLin-KC2-DMA (2,2-dilinoleyl-4-(2-dimethylaminoethyl)-[1,3]-dioxolane) [13], and DLin-MC3-DMA [14], which are 100-fold and 1000-fold more potent, respectively, in silencing of a hepatic gene (Factor VII) in comparison to the previous generation lipid DLin-DMA (1,2-dilinoleyloxy- $N, N$-dimethyl-3-aminopropane) [29]. The $\mathrm{ED}_{50}$ (median effective dose) for LNP containing DLin-MC3-DMA to silence Factor VII in mice and TTR in non-human primates was $0.005 \mathrm{mg} / \mathrm{kg}$ and $0.03 \mathrm{mg} / \mathrm{kg}$, respectively [14]. One of the key findings from these studies was the optimum lipid $\mathrm{pK}_{\mathrm{a}}$ value of $6.2-6.5$ as a dominating factor in determining hepatic gene-silencing activity in vivo. DLin-MC3-DMA, having a pKa of 6.44 , is currently the most active ionizable lipid being used in clinical trials.

In hopes of further promoting biocompatibility, a novel generation of ionizable amino lipids was recently synthesized with biodegradable ester functionalities in the acyl chains [30]. These novel lipids were well tolerated by the animals and rapidly eliminated from plasma and tissues. Importantly, they exhibited excellent potencies $\left(\mathrm{ED}_{50}<0.01 \mathrm{mg} / \mathrm{kg}\right.$ in mice, similar to that of DLin-MC3-DMA) in both rodent and non-human primate models. Although the relevance of these findings to human treatment remains to be proven, these preclinical results for biodegradable LNP siRNA formulations show great promise for clinical applications. 


\section{Steric Barrier: Stability and Circulation Lifetime}

Unprotected lipid-based delivery systems are rapidly cleared by the mononuclear phagocyte system [31,32]. In order to overcome this, hydrophilic polyethylene glycol has been widely used to coat lipid-based delivery systems, which prevents aggregation during the formulation process as well as provide "stealth" like characteristics post $i . v$. administration. The optimized PEG-lipid characteristics in current LNP siRNA systems were largely derived from earlier work on PEGylated liposomes [33-35] and stabilized plasmid or antisense lipid particles (SPLP or SALP). It was found that SPLP or SALP with PEG-ceramide that have $\mathrm{C}_{20}$ anchors $\left(\mathrm{PEG}-\mathrm{CerC}_{20}\right)$ exhibited poor transfection efficiency in cultured cells compared to its shorter anchor counterparts such as $\mathrm{PEG}-\mathrm{CerC}_{14}$ or $\mathrm{PEG}-\mathrm{CerC}$ [36-38]. The length of the ceramide lipid anchor dictated how long the PEG-lipid remained associated with the LNP. PEG-lipids with longer acyl anchors remain associated with the LNP longer, thus preventing LNP interaction with cells and subsequently with the endosomal membranes. They also provide the LNP with a more durable coat, which extends circulation lifetime [39,40]. Consistent findings were observed when PEG-ceramides were replaced with PEG-succinoyl-diacylglycerols (PEG-s-DAG) that have various acyl anchor lengths [41]. Due to the ease of synthesis and purification, PEG-s-DAG replaced PEG-Cer in later SPLP formulations and long acyl chain PEG-s-DAG such as PEG-s-distearoylglycerol (PEG-s-DSG) were used to increase circulation lifetime in the hope of exploiting the enhanced permeability and retention effect at tumor sites.

Unfortunately, despite being efficacious and non-toxic after a single bolus injection, PEGylated LNP with long acyl anchors (PEG-DSPE (1,2-distearoyl-sn-glycero-3-phosphoethanolamine), PEG-s-DSG, or PEG-CerC 20 ) were rapidly cleared upon repeated administration as a result of a robust immune response to the PEG component $[42,43]$. The use of rapidly dissociated shorter anchor PEG-lipids such as PEG-s-DMG or PEG-CerC 14 however, mitigated this immunogenic response. Coincidentally, it was also observed that LNP containing PEG-s-DAG progressively lost the PEG moiety due to its succinate linker leading to particle aggregation and a reduced shelf-life. In response to this, the succinate linker was replaced with a carbamate linker to confer improved chemical stability without affecting efficacy [44].

\section{Cellular Uptake: Endogenous and Exogenous Targeting Ligands}

Neutral liposomes have been shown to bind to proteins in serum, exchange components with lipoproteins and acquire factors that can potentially target them to specific cell types [45,46]. In particular, they interact with apolipoprotein (Apo) E and A-I [47,48]. ApoE, but not ApoA-I or ApoA-IV, was further found to enhance uptake of neutral liposomes in HepG2 cells and primary hepatocytes [49]. The role of ApoE in LNP uptake into hepatocytes was confirmed in vivo using ApoE-deficient mice [50]. The authors demonstrated that LNP were cleared more slowly from the circulation and were taken up by hepatocytes at least 20-fold less in ApoE-deficient mice than in wild-type animals. Similarly, LNP siRNA systems containing ionizable lipids require ApoE for activity [51]. Silencing of Factor VII was compromised in mice lacking ApoE or low-density lipoprotein (LDL) receptor, suggesting that ApoE acts as an endogenous ligand for LNP siRNA systems that facilitate uptake into hepatocytes via the LDL receptor. 
When endogenous ligands are not available, exogenous ligands can be used to enhance uptake of LNP siRNA systems in target cells. Exogenous ligands such as antibodies, antibody fragments and peptides have been widely used in the field of liposome technology [52-55]. However, they are expensive and difficult to manufacture, as well as potentially immunogenic. In contrast, small molecule ligands conjugated to the distal end of PEG-lipids are simple to synthesize and can be formulated into LNP in a straightforward manner (Figure 2) [51,56,57]. N-Acetylgalactosamine (GalNAc), which binds with high affinity to the asialoglycoprotein receptor (ASGPR) found on hepatocytes, have been shown to rescue gene-silencing activity of LNP siRNA systems in ApoE deficient mice [51]. Other exogenous small molecule ligands have shown utility in non-hepatic cells. Anisamide, which interacts with sigma receptors, increases delivery and activity of siRNA containing nanoparticles in lung tumors and metastases [56,58,59]. Furthermore, strophanthidin, a cardiac glycoside that binds to the ubiquitously expressed cell surface receptor $\mathrm{Na}^{+} / \mathrm{K}^{+}$ATPase, has been shown to enhance delivery of LNP siRNA to various cell types originating from the ovary, breast, pancreas, lung and prostate [57]. While the delivery of LNP using a promiscuous ligand may be non-specific, target specificity can be conferred using siRNA against disease-causing genes that are expressed in specific tissues.

\section{Concluding Remarks}

Using LNP as the delivery platform for siRNA has undoubtedly increased the potential of siRNA as therapeutics. This is evidenced by five LNP siRNA formulations for various liver-related diseases in clinical trials and more in the pipeline. The increased understanding of individual formulation components and their composition, LNP structure as well as formulation methods have led to the development of increasingly potent LNP siRNA systems. The success of LNP in hepatic applications is due, at least in part, to the liver's ideal physiology, notably being highly perfused with fenestrated endothelium. Other contributing factors include the optimum $\mathrm{pK}_{\mathrm{a}}$ of the ionizable lipid, dissociable PEG-lipid, and association of LNP with endogenous ligand ApoE.

A number of challenges remain to be overcome for LNP siRNA to be used as therapeutics in a broad range of diseases. The existing LNP siRNA systems for liver applications have to be modified to extend their utility for non-hepatic tissues such as distal tumors. Novel small molecule targeting ligands will be required to facilitate LNP uptake into these non-hepatic tissues, especially when the siRNA is not tissue-specific. In order to reach tumor cores or tumors with poor vascularization, small LNP may be useful $[60,61]$. LNP siRNA systems as small as $25 \mathrm{~nm}$ can be made using microfluidic micromixing technology [22]; however, the relatively small siRNA payload may compromise activity and alternative methods to increase potency will have to be explored. Furthermore, LNP composition will likely require modifications for other routes of administration such as intraperitoneal, subcutaneous, intranasal or topical.

\section{Acknowledgments}

The authors would like to acknowledge the Canadian Institutes of Health Research (CIHR) grants FRN 111627 and 86587, Alnylam Pharmaceuticals and Tekmira Pharmaceuticals for supporting this 
work. Karen Lam is gratefully acknowledged for editing the manuscript and illustrating the schematic. Josh Zaifman is gratefully thanked for providing the lipid structures.

\section{Conflicts of Interest}

The authors declare no conflict of interest.

\section{References}

1. Novina, C.D.; Sharp, P.A. The rnai revolution. Nature 2004, 430, 161-164.

2. Vaishnaw, A.K.; Gollob, J.; Gamba-Vitalo, C.; Hutabarat, R.; Sah, D.; Meyers, R.; de Fougerolles, T.; Maraganore, J. A status report on rnai therapeutics. Silence 2010, 1, 14.

3. McManus, M.T.; Sharp, P.A. Gene silencing in mammals by small interfering rnas. Nat. Rev. Genet. 2002, 3, 737-747.

4. Miele, E.; Spinelli, G.P.; di Fabrizio, E.; Ferretti, E.; Tomao, S.; Gulino, A. Nanoparticle-based delivery of small interfering RNA: Challenges for cancer therapy. Int. J. Nanomed. 2012, 7, 3637-3657.

5. De Fougerolles, A.; Vornlocher, H.P.; Maraganore, J.; Lieberman, J. Interfering with disease: A progress report on siRNA-based therapeutics. Nat. Rev. Drug Discov. 2007, 6, 443-453.

6. Whitehead, K.A.; Langer, R.; Anderson, D.G. Knocking down barriers: Advances in sirna delivery. Nat. Rev. Drug Discov. 2009, 8, 129-138.

7. Manoharan, M.; Akinc, A.; Pandey, R.K.; Qin, J.; Hadwiger, P.; John, M.; Mills, K.; Charisse, K.; Maier, M.A.; Nechev, L.; et al. Unique gene-silencing and structural properties of 2'-fluoro-modified sirnas. Angew. Chem. Int. Ed. Engl. 2011, 50, 2284-2288.

8. Manoharan, M. RNA interference and chemically modified small interfering RNAs. Curr. Opin. Chem. Biol. 2004, 8, 570-579.

9. Engels, J.W. Gene silencing by chemically modified sirnas. New Biotechnol. 2013, 30, 302-307.

10. Soutschek, J.; Akinc, A.; Bramlage, B.; Charisse, K.; Constien, R.; Donoghue, M.; Elbashir, S.; Geick, A.; Hadwiger, P.; Harborth, J.; et al. Therapeutic silencing of an endogenous gene by systemic administration of modified sirnas. Nature 2004, 432, 173-178.

11. Allen, T.M.; Cullis, P.R. Liposomal drug delivery systems: From concept to clinical applications. Adv. Drug Deliv. Rev. 2012, 65, 36-48.

12. Daka, A.; Peer, D. RNAi-based nanomedicines for targeted personalized therapy. Adv. Drug Deliv. Rev. 2012, 64, 1508-1521.

13. Semple, S.C.; Akinc, A.; Chen, J.; Sandhu, A.P.; Mui, B.L.; Cho, C.K.; Sah, D.W.; Stebbing, D.; Crosley, E.J.; Yaworski, E.; et al. Rational design of cationic lipids for siRNA delivery. Nat. Biotechnol. 2010, 28, 172-176.

14. Jayaraman, M.; Ansell, S.M.; Mui, B.L.; Tam, Y.K.; Chen, J.; Du, X.; Butler, D.; Eltepu, L.; Matsuda, S.; Narayanannair, J.K.; et al. Maximizing the potency of sirna lipid nanoparticles for hepatic gene silencing in vivo. Angew. Chem. Int. Ed. Engl. 2012, 51, 8529-8533.

15. Huang, L.; Liu, Y. In vivo delivery of rnai with lipid-based nanoparticles. Annu. Rev. Biomed. Eng. 2011, 13, 507-530.

16. Burnett, J.C.; Rossi, J.J.; Tiemann, K. Current progress of siRNA/shRNA therapeutics in clinical trials. Biotechnol. J. 2011, 6, 1130-1146. 
17. Barros, S.A.; Gollob, J.A. Safety profile of RNAi nanomedicines. Adv. Drug Deliv. Rev. 2012, 64, 1730-1737.

18. Tabernero, J.; Shapiro, G.I.; LoRusso, P.M.; Cervantes, A.; Schwartz, G.K.; Weiss, G.J.; Paz-Ares, L.; Cho, D.C.; Infante, J.R.; Alsina, M.; et al. First-in-humans trial of an RNA interference therapeutic targeting vegf and ksp in cancer patients with liver involvement. Cancer Discov. 2013, 3, 406-417.

19. Alabi, C.; Vegas, A.; Anderson, D. Attacking the genome: Emerging sirna nanocarriers from concept to clinic. Curr. Opin. Pharmacol. 2012, 12, 427-433.

20. Crawford, R.; Dogdas, B.; Keough, E.; Haas, R.M.; Wepukhulu, W.; Krotzer, S.; Burke, P.A.; Sepp-Lorenzino, L.; Bagchi, A.; Howell, B.J. Analysis of lipid nanoparticles by cryo-em for characterizing siRNA delivery vehicles. Int. J. Pharm. 2011, 403, 237-244.

21. Leung, A.K.; Hafez, I.M.; Baoukina, S.; Belliveau, N.M.; Zhigaltsev, I.V.; Afshinmanesh, E.; Tieleman, D.P.; Hansen, C.L.; Hope, M.J.; Cullis, P.R. Lipid nanoparticles containing sirna synthesized by microfluidic mixing exhibit an electron-dense nanostructured core. J. Phys. Chem. C Nanomater. Interfaces 2012, 116, 18440-18450.

22. Belliveau, N.M.; Huft, J.; Lin, P.J.C.; Chen, S.; Leung, A.K.K.; Leaver, T.J.; Wild, A.W.; Lee, J.B.; Taylor, R.J.; Tam, Y.K.; et al. Microfluidic synthesis of highly potent limit-size lipid nanoparticles for in vivo delivery of sirna. Mol. Ther. Nucleic Acids 2012, 1, e37.

23. Zhigaltsev, I.V.; Maurer, N.; Edwards, K.; Karlsson, G.; Cullis, P.R. Formation of drug-arylsulfonate complexes inside liposomes: A novel approach to improve drug retention. J. Control. Release 2006, 110, 378-386.

24. Semple, S.C.; Klimuk, S.K.; Harasym, T.O.; Dos Santos, N.; Ansell, S.M.; Wong, K.F.; Maurer, N.; Stark, H.; Cullis, P.R.; Hope, M.J.; et al. Efficient encapsulation of antisense oligonucleotides in lipid vesicles using ionizable aminolipids: Formation of novel small multilamellar vesicle structures. Biochim. Biophys. Acta 2001, 1510, 152-166.

25. Maurer, N.; Wong, K.F.; Stark, H.; Louie, L.; McIntosh, D.; Wong, T.; Scherrer, P.; Semple, S.C.; Cullis, P.R. Spontaneous entrapment of polynucleotides upon electrostatic interaction with ethanol-destabilized cationic liposomes. Biophys. J. 2001, 80, 2310-2326.

26. Hafez, I.M.; Maurer, N.; Cullis, P.R. On the mechanism whereby cationic lipids promote intracellular delivery of polynucleic acids. Gene Ther. 2001, 8, 1188-1196.

27. Xu, Y.; Szoka, F.C., Jr. Mechanism of DNA release from cationic liposome/DNA complexes used in cell transfection. Biochemistry 1996, 35, 5616-5623.

28. Bailey, A.L.; Cullis, P.R. Modulation of membrane fusion by asymmetric transbilayer distributions of amino lipids. Biochemistry 1994, 33, 12573-12580.

29. Heyes, J.; Palmer, L.; Bremner, K.; MacLachlan, I. Cationic lipid saturation influences intracellular delivery of encapsulated nucleic acids. J. Control. Release 2005, 107, 276-287.

30. Maier, M.A.; Jayaraman, M.; Matsuda, S.; Liu, J.; Barros, S.; Querbes, W.; Tam, Y.K.; Ansell, S.M.; Kumar, V.; Qin, J.; et al. Biodegradable lipids enabling rapidly eliminated lipid nanoparticles for systemic delivery of RNAi therapeutics. Mol. Ther. 2013, 21, 1570-1578.

31. Allen, T.M. The use of glycolipids and hydrophilic polymers in avoiding rapid uptake of liposomes by the mononuclear phagocyte system. Adv. Drug Deliv. Rev. 1994, 13, 285-309.

32. Ishida, T.; Harashima, H.; Kiwada, H. Liposome clearance. Biosci. Rep. 2002, 22, 197-224. 
33. Klibanov, A.L.; Maruyama, K.; Torchilin, V.P.; Huang, L. Amphipathic polyethyleneglycols effectively prolong the circulation time of liposomes. FEBS Lett. 1990, 268, 235-237.

34. Webb, M.S.; Saxon, D.; Wong, F.M.; Lim, H.J.; Wang, Z.; Bally, M.B.; Choi, L.S.; Cullis, P.R.; Mayer, L.D. Comparison of different hydrophobic anchors conjugated to poly(ethylene glycol): Effects on the pharmacokinetics of liposomal vincristine. Biochim. Biophys. Acta 1998, 1372, 272-282.

35. Woodle, M.C.; Lasic, D.D. Sterically stabilized liposomes. Biochim. Biophys. Acta 1992, 1113, 171-199.

36. Wheeler, J.J.; Palmer, L.; Ossanlou, M.; MacLachlan, I.; Graham, R.W.; Zhang, Y.P.; Hope, M.J.; Scherrer, P.; Cullis, P.R. Stabilized plasmid-lipid particles: Construction and characterization. Gene Ther. 1999, 6, 271-281.

37. Mok, K.W.; Lam, A.M.; Cullis, P.R. Stabilized plasmid-lipid particles: Factors influencing plasmid entrapment and transfection properties. Biochim. Biophys. Acta 1999, 1419, 137-150.

38. Song, L.Y.; Ahkong, Q.F.; Rong, Q.; Wang, Z.; Ansell, S.; Hope, M.J.; Mui, B. Characterization of the inhibitory effect of PEG-lipid conjugates on the intracellular delivery of plasmid and antisense DNA mediated by cationic lipid liposomes. Biochim. Biophys. Acta 2002, 1558, 1-13.

39. Monck, M.A.; Mori, A.; Lee, D.; Tam, P.; Wheeler, J.J.; Cullis, P.R.; Scherrer, P. Stabilized plasmid-lipid particles: Pharmacokinetics and plasmid delivery to distal tumors following intravenous injection. J. Drug Target. 2000, 7, 439-452.

40. Tam, P.; Monck, M.; Lee, D.; Ludkovski, O.; Leng, E.C.; Clow, K.; Stark, H.; Scherrer, P.; Graham, R.W.; Cullis, P.R. Stabilized plasmid-lipid particles for systemic gene therapy. Gene Ther. 2000, 7, 1867-1874.

41. Ambegia, E.; Ansell, S.; Cullis, P.; Heyes, J.; Palmer, L.; MacLachlan, I. Stabilized plasmid-lipid particles containing PEG-diacylglycerols exhibit extended circulation lifetimes and tumor selective gene expression. Biochim. Biophys. Acta 2005, 1669, 155-163.

42. Judge, A.; McClintock, K.; Phelps, J.R.; Maclachlan, I. Hypersensitivity and loss of disease site targeting caused by antibody responses to pegylated liposomes. Mol. Ther. 2006, 13, 328-337.

43. Semple, S.C.; Harasym, T.O.; Clow, K.A.; Ansell, S.M.; Klimuk, S.K.; Hope, M.J. Immunogenicity and rapid blood clearance of liposomes containing polyethylene glycol-lipid conjugates and nucleic acid. J. Pharmacol. Exp. Ther. 2005, 312, 1020-1026.

44. Heyes, J.; Hall, K.; Tailor, V.; Lenz, R.; MacLachlan, I. Synthesis and characterization of novel poly(ethylene glycol)-lipid conjugates suitable for use in drug delivery. J. Control. Release 2006, 112, 280-290.

45. Chonn, A.; Semple, S.C.; Cullis, P.R. Association of blood proteins with large unilamellar liposomes in vivo. Relation to circulation lifetimes. J. Biol. Chem. 1992, 267, 18759-18765.

46. Cullis, P.R.; Chonn, A.; Semple, S.C. Interactions of liposomes and lipid-based carrier systems with blood proteins: Relation to clearance behaviour in vivo. Adv. Drug Deliv. Rev. 1998, 32, 3-17.

47. Mendez, A.J.; He, J.L.; Huang, H.S.; Wen, S.R.; Hsia, S.L. Interaction of rabbit lipoproteins and red blood cells with liposomes of egg yolk phospholipids. Lipids 1988, 23, 961-967. 
48. Rensen, P.C.; Schiffelers, R.M.; Versluis, A.J.; Bijsterbosch, M.K.; van Kuijk-Meuwissen, M.E.; van Berkel, T.J. Human recombinant apolipoprotein e-enriched liposomes can mimic low-density lipoproteins as carriers for the site-specific delivery of antitumor agents. Mol. Pharmacol. 1997, $52,445-455$.

49. Bisgaier, C.L.; Siebenkas, M.V.; Williams, K.J. Effects of apolipoproteins A-IV and A-I on the uptake of phospholipid liposomes by hepatocytes. J. Biol. Chem. 1989, 264, 862-866.

50. Yan, X.; Kuipers, F.; Havekes, L.M.; Havinga, R.; Dontje, B.; Poelstra, K.; Scherphof, G.L.; Kamps, J.A. The role of apolipoprotein e in the elimination of liposomes from blood by hepatocytes in the mouse. Biochem. Biophys. Res. Commun. 2005, 328, 57-62.

51. Akinc, A.; Querbes, W.; De, S.; Qin, J.; Frank-Kamenetsky, M.; Jayaprakash, K.N.; Jayaraman, M.; Rajeev, K.G.; Cantley, W.L.; Dorkin, J.R.; et al. Targeted delivery of rnai therapeutics with endogenous and exogenous ligand-based mechanisms. Mol. Ther. 2010, 18, 1357-1364.

52. Sapra, P.; Allen, T.M. Ligand-targeted liposomal anticancer drugs. Progr. Lipid Res. 2003, 42, 439-462.

53. Cressman, S.; Dobson, I.; Lee, J.B.; Tam, Y.Y.; Cullis, P.R. Synthesis of a labeled RGD-lipid, its incorporation into liposomal nanoparticles, and their trafficking in cultured endothelial cells. Bioconjug. Chem. 2009, 20, 1404-1411.

54. Allen, T.M.; Sapra, P.; Moase, E.; Moreira, J.; Iden, D. Adventures in targeting. J. Liposome Res. 2002, 12, 5-12.

55. Di Paolo, D.; Ambrogio, C.; Pastorino, F.; Brignole, C.; Martinengo, C.; Carosio, R.; Loi, M.; Pagnan, G.; Emionite, L.; Cilli, M.; et al. Selective therapeutic targeting of the anaplastic lymphoma kinase with liposomal sirna induces apoptosis and inhibits angiogenesis in neuroblastoma. Mol. Ther. 2011, 19, 2201-2212.

56. Li, S.D.; Huang, L. Targeted delivery of antisense oligodeoxynucleotide and small interference RNA into lung cancer cells. Mol. Pharm. 2006, 3, 579-588.

57. Tam, Y.Y.C.; Chen, S.; Zaifman, J.; Tam, Y.K.; Lin, P.J.C.; Ansell, S.; Roberge, M.; Ciufolini, M.A.; Cullis, P.R. Small molecule ligands for enhanced intracellular delivery of lipid nanoparticle formulations of siRNA. Nanomed. Nanotechnol. Biol. Med. 2013, 9, 665-674.

58. Li, S.D.; Chono, S.; Huang, L. Efficient oncogene silencing and metastasis inhibition via systemic delivery of siRNA. Mol. Ther. 2008, 16, 942-946.

59. Chen, Y.; Sen, J.; Bathula, S.R.; Yang, Q.; Fittipaldi, R.; Huang, L. Novel cationic lipid that delivers siRNA and enhances therapeutic effect in lung cancer cells. Mol. Pharm. 2009, 6, 696-705.

60. Cabral, H.; Matsumoto, Y.; Mizuno, K.; Chen, Q.; Murakami, M.; Kimura, M.; Terada, Y.; Kano, M.R.; Miyazono, K.; Uesaka, M.; et al. Accumulation of sub-100 nm polymeric micelles in poorly permeable tumours depends on size. Nat. Nanotechnol. 2011, 6, 815-823.

61. Huo, S.; Ma, H.; Huang, K.; Liu, J.; Wei, T.; Jin, S.; Zhang, J.; He, S.; Liang, X.J. Superior penetration and retention behavior of $50 \mathrm{~nm}$ gold nanoparticles in tumors. Cancer Res. 2013, 73, $319-330$.

(C) 2013 by the authors; licensee MDPI, Basel, Switzerland. This article is an open access article distributed under the terms and conditions of the Creative Commons Attribution license (http://creativecommons.org/licenses/by/3.0/). 